\title{
Surgical Strategy Using Endoscopy to Minimize Fetal Surgical Risks in Treating a Pregnant Woman with Herniated Lumbar Disc Disease
}

\author{
Byeong Sam Choi ${ }^{1} \quad$ II Choi $^{2}$ Sungjoon Lee ${ }^{1}$ \\ ${ }^{1}$ Department of Neurosurgery, Inje University Haeundae Paik \\ Hospital, Busan, Korea \\ 2 Department of Neurosurgery, Dongtan Sacred Heart Hospital, \\ Hallym University, Gyeonggi-do, Korea \\ Indian J Neurosurg 2016;5:51-54.
}

\author{
Address for correspondence Sungjoon Lee, MD, Department of \\ Neurosurgery, Inje University, Haeundae Paik Hospital, 875 \\ Haeundae-ro, Haeundae-gu, Busan 612-896, Korea \\ (e-mail: potata98@naver.com).
}

\begin{abstract}
Pregnancy poses a special clinical challenge in surgical treatments for spinal diseases. Here we present the case of a 30-year-old woman with a ruptured lumbar disc on the right side of L5-S1. The patient was 18 weeks pregnant. Conservative managements for 3 weeks had failed to relieve her pain. We performed interlaminar percutaneous endoscopic lumbar discectomy (PELD) using spinal anesthesia. The left lateral decubitus position was chosen to minimize abdominal compression. Preoperative magnetic resonance imaging (MRI) taken after attaching few fiducial markers at patient's mid-lower back was used to secure the correct surgical level without X-rays. The surgery was successful, and the patient's pain score on a visual analogue scale was reduced from $9 / 10$ to $3 / 10$. Ultrasonography was used to confirm that the baby was

Keywords

- endoscopic discectomy

- pregnancy

- lumbar disc herniation healthy postoperatively. PELD causes less pain, and dose not require general anesthesia, which imposes a higher risk of fetal distress than regional anesthesia. Combination of the interlaminar approach of accessing from the midline and a marking MRI permitted PELD without X-ray imaging. The relative ease of performing surgery on either side of the lateral decubitus position makes PELD more feasible for pregnant patients.
\end{abstract}

\section{Introduction}

The incidence of symptomatic lumbar disc herniation during pregnancy is rare and occurs in only 1 in approximately 10,000 pregnancies. ${ }^{1,2}$ Conservative management should be the first treatment. However, surgical treatment is considered for patients with a medical emergency such as cauda equina syndrome or patients with intractable pain despite proper conservative treatment.

The literature clearly demonstrates that most surgeries can be performed safely at any stage of pregnancy. ${ }^{2-9}$ However, the use of medications, anesthesia, image modalities involving X-rays, and surgical position all pose risks to both the fetus and mother. ${ }^{3}$ We report a surgically treated case of lumbar disc herniation using endoscopy in the second trimester of pregnancy. This case is presented to propose a feasible surgical strategy using endoscope that minimizes the surgical risks in pregnancy.

\section{Case Report}

A 30-year-old woman presented at 18-week gestation in her second pregnancy with a 1-week history of lower back pain and radiating pain of the right posterior leg. On neurologic examination, hypoesthesia in her right posterior thigh and calf was presented. The straight leg raising test of the right received

August 10, 2015

accepted

November 23, 2015

published online

February 19, 2016
DOI http://dx.doi.org/

10.1055/s-0036-1572374. ISSN 2277-954X.
(C) 2016 Neurological Surgeons' Society of India

License terms

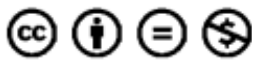




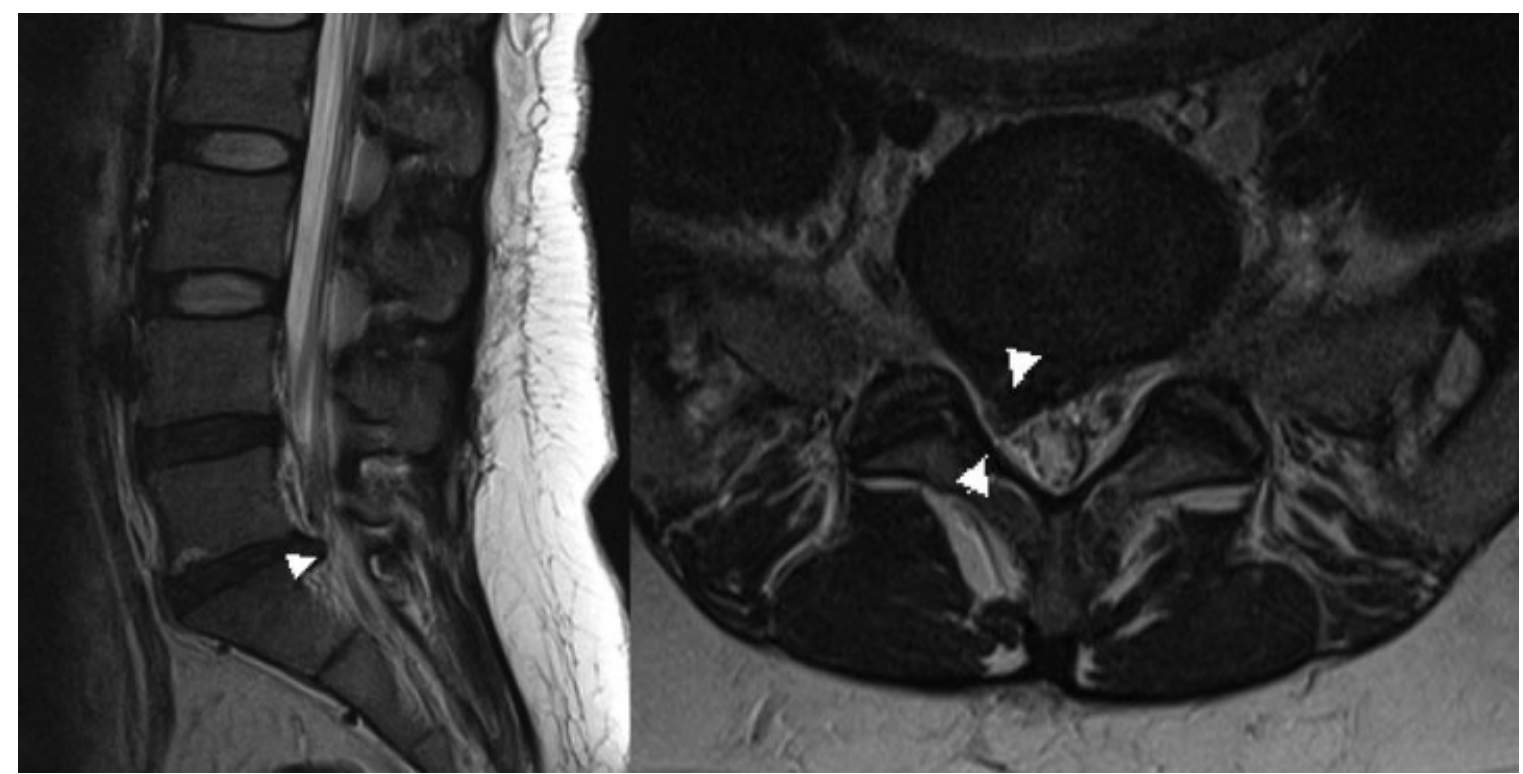

Fig. 1 Sagittal (left) and axial (right) T2-weighted magnetic resonance images showed a right-sided, paracentral disc herniation at L5-S1 level (white triangles).

leg was restricted to 20 degrees. There was no motor weakness of the lower extremities found. Magnetic resonance imaging (MRI) showed a right-sided, paracentral disc herniation at L5-S1 that was compressing the right S1 nerve root (-Fig. 1). The patient was first treated conservatively for 3 weeks. Oral medication, ultrasoundguided caudal block, and continuous epidural patientcontrolled analgesia (PCA) were used. However, these treatments failed to relieve her pain. The patient wanted surgical treatment to relieve her pain immediately, but refused any procedure potentially harmful to her baby.

We planned L5-S1 interlaminar percutaneous endoscopic lumbar discectomy (PELD) under spinal anesthesia. The left lateral decubitus position was chosen for the patient's and baby's safety during surgery. For the spinal anesthesia, the patient received $8 \mathrm{mg}$ of $0.5 \%$ bupivacaine injected intrathecally through the L4-5 level. To establish the operating level without X-rays, we performed a marking MRI that consisted of T2weighted sagittal images after attaching three fiducial markers at the patient's mid-back area. The markers started at the iliac crest level, which is the bony landmark of the L4-5 level. The markers were attached at intervals of $1 \mathrm{~cm}$ in the caudal direction and covered the L5-S1 level (-Fig. 2). We used marking MRI, guidance of bony landmarks, and feeling the lamina bone through a dilator and working sleeve. It was not difficult to secure the correct surgical level using this combination strategy. Skin entry was the point between the middle and lower fiducial markers (white arrow, -Fig. 2) where the L5-S1 interlaminar space was located vertically below. Procedures requiring intraoperative X-rays such as discography were not performed. Fetal heart monitoring was not conducted during the operation because the systemic effect of spinal anesthesia was minimal. The ruptured disc was removed under full endoscopic view, and the operation was uneventful. A postoperative MRI revealed the ruptured disc was removed (-Fig. $\mathbf{3}$ ). The patient preoperative visual analogue scale (VAS) pain score of the leg was 9/10. The immediate postoperative VAS pain score improved to $3 / 10$. An ultrasound examination was performed to determine the status of the fetus 2 days after the surgery. The obstetrician confirmed that the baby was healthy.

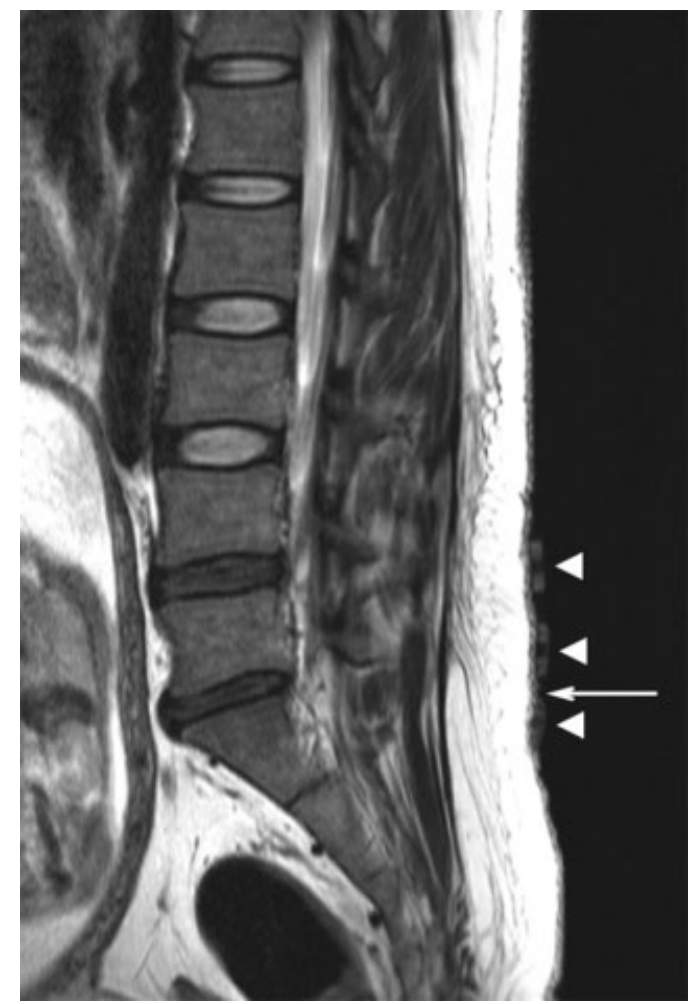

Fig. 2 Marking magnetic resonance images consisted of only T2weighted sagittal images taken after attaching fiducial markers on the patient's lower back (white triangles). To enter the L5-S1 interlaminar space vertically from the skin, a mid-point between middle and lower fiducial marker (marked by white arrow) was selected for the skin entry point. 


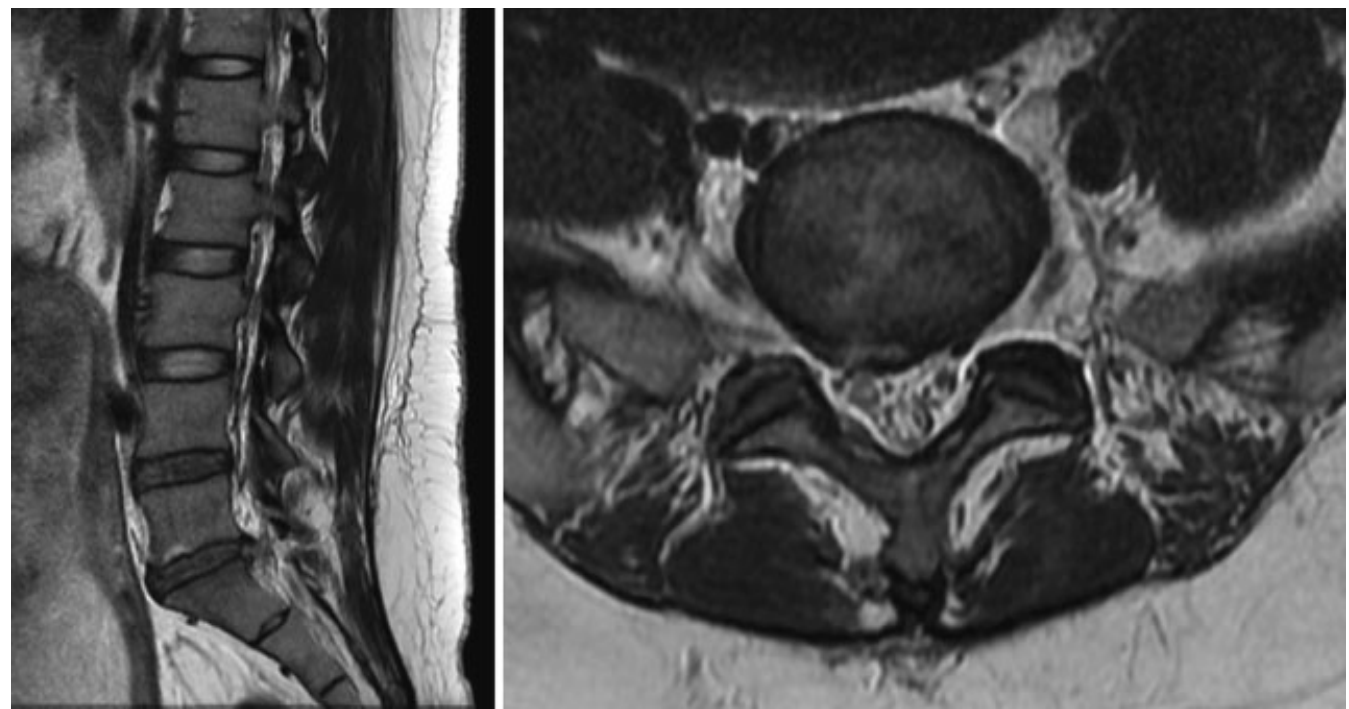

Fig. 3 Postoperative sagittal (left) and axial (right) T2-weighted magnetic resonance images showed the removed herniated disc at L5-S1 level.

\section{Discussion}

Although the incidence of symptomatic lumbar disc herniation in pregnancy is rare, there are several reports demonstrating successful surgical treatment. ${ }^{2-9}$ There are roughly four concerns for fetal safety regarding lumbar disc surgery: medications, anesthesia, surgical position, and diagnostic measures involving specific tools that use radiation.

Medical managements of patients in pregnancy are troublesome because there are a limited number of analgesics that can be used safely. Most nonsteroidal anti-inflammatory drugs and muscle relaxants are Food and Drug Administration (FDA) category C and D, and the potential for congenital anomalies has been reported. ${ }^{3,10}$ Ultrasound-guided epidural injection or epidural PCA could be a safe and effective modality. However, these methods do not always guarantee successful pain control, as shown in our case. In such cases, surgery could be an alternative treatment option because it can provide immediate and definite pain relief.

Pregnancy at any stage is not a contraindication for the administration of general anesthesia., ${ }^{11,12}$ However, it is clear that the fetus may be influenced by anesthetic effects caused by the perfusion and placental transfer of depressant drugs. ${ }^{11}$ Therefore, it is preferable to choose regional anesthesia such as epidural or spinal anesthesia instead of general anesthesia. ${ }^{2,3}$ Considering the short operation time and less procedure-related pain, PELD under regional anesthesia could be an excellent modality for lumbar disc surgery during pregnancy.

In addition, PELD is feasible because it can be performed easily in either the prone or lateral decubitus position. Surgery can be performed during the first and early part of the second trimester in the prone position. However, left lateral decubitus position is indicated during the second and third trimesters to minimize aortocaval compression. ${ }^{3}$
Microdiskectomy in the left lateral decubitus position is possible and several authors have reported successful experiences. ${ }^{3,11}$ However, this is a technically challenging surgery. The instrument handling of interlaminar PELD in the lateral decubitus position is quite similar to that of transforaminal PELD in the prone position. In addition, the endoscopic view is not different from that of a usual interlaminar PELD. One thing surgeons should be aware of is that the surgical position is sometimes determined regardless of the location of the lesion (left lateral decubitus position in late second and third trimesters) and the operating view of interlaminar PELD is rotated 180 degrees if the lesion is located on the upper side of a patient in the lateral decubitus position, that is, right-side disc herniation in left lateral decubitus position or vice versa. In our case, we operated on right-side disc herniation in the left lateral decubitus position. The discectomy was performed without any difficulties using a rotating endoscope maneuver.

One major barrier in the application of PELD technique during pregnancy despite of all the advantages is high intraoperative radiation exposure. To avoid intraoperative Xrays, we used a preoperative marking MRI consisting of only T2-weighted sagittal images because it is safe on the developing fetus. $2,3,13,14$ The lamina bone can be easily felt with dilators and working sleeves, and we readily identified interlaminar window, and if we approach the interlaminar window of the index level vertically from the skin, the probability of entering the wrong level is low because a change of at least 30-degree inclination in the working sleeve to the cephalad or caudal direction is required to access one level above or below the index level. We believe that the transforaminal approach is not appropriate in lumbar discectomy during pregnancy. In contrast to the interlaminar approach, the exact entry of Kambin's triangle is essential to avoid exiting root injury, and this cannot be achieved without the use of intraoperative X-ray. 
54 PELD Technique to Minimize Surgical Risk in a Pregnant Woman Choi et al.

\section{Conclusion}

PELD causes less pain and dose not require general anesthesia, which imposes a higher risk of fetal distress than regional anesthesia. The interlaminar approach of accessing from the midline and a marking MRI permitted PELD without X-ray imaging. The relative ease of performing surgery on either side of the lateral decubitus position makes PELD more feasible for pregnant patients.

\section{References}

1 LaBan MM, Rapp NS, von Oeyen P, Meerschaert JR. The lumbar herniated disk of pregnancy: a report of six cases identified by magnetic resonance imaging. Arch Phys Med Rehabil 1995;76(5): 476-479

2 Brown MD, Levi AD. Surgery for lumbar disc herniation during pregnancy. Spine 2001;26(4):440-443

3 Han IH, Kuh SU, Kim JH, et al. Clinical approach and surgical strategy for spinal diseases in pregnant women: a report of ten cases. Spine 2008;33(17):E614-E619

4 Abou-Shameh MA, Dosani D, Gopal S, McLaren AG. Lumbar discectomy in pregnancy. Int J Gynaecol Obstet 2006;92(2):167-169

5 Fahy UM, Oni M, Findlay D, Sell P. Surgical management of herniated lumbar disc in pregnancy. J Obstet Gynaecol 1998; 18(6):544-545
6 Garmel SH, Guzelian GA, D’Alton JG, D’Alton ME. Lumbar disk disease in pregnancy. Obstet Gynecol 1997;89(5 Pt 2): $821-822$

7 Kathirgamanathan A, Jardine AD, Levy DM, Grevitt MP. Lumbar disc surgery in the third trimester-with the fetus in utero. Int J Obstet Anesth 2006;15(2):181-182

$8 \mathrm{Kim}$ HS, Kim SW, Lee SM, Shin H. Endoscopic discectomy for the cauda equina syndrome during third trimester of pregnancy. J Korean Neurosurg Soc 2007;42(5):419-420

9 Hakan T. Lumbar disk herniation presented with cauda equina syndrome in a pregnant woman. J Neurosci Rural Pract 2012; 3(2):197-199

10 Ofori B, Oraichi D, Blais L, Rey E, Bérard A. Risk of congenital anomalies in pregnant users of non-steroidal anti-inflammatory drugs: a nested case-control study. Birth Defects Res B Dev Reprod Toxicol 2006;77(4):268-279

11 Iyilikçi L, Erbayraktar S, Tural AN, Celik M, Sannav S. Anesthetic management of lumbar discectomy in a pregnant patient. J Anesth 2004;18(1):45-47

12 Mazze RI, Källén B. Reproductive outcome after anesthesia and operation during pregnancy: a registry study of 5405 cases. Am J Obstet Gynecol 1989;161(5):1178-1185

13 Ostgaard HC, Andersson GB, Karlsson K. Prevalence of back pain in pregnancy. Spine 1991;16(5):549-552

14 Weinreb JC, Wolbarsht LB, Cohen JM, Brown CE, Maravilla KR. Prevalence of lumbosacral intervertebral disk abnormalities on MR images in pregnant and asymptomatic nonpregnant women. Radiology 1989;170(1 Pt 1):125-128 